\title{
An order-theoretic approach to dynamic programming: an exposition
}

\author{
Takashi Kamihigashi
}

Received: 15 November 2013 / Accepted: 20 November 2013 / Published online: 12 December 2013 (C) SAET 2013

\begin{abstract}
In this note, we discuss an order-theoretic approach to dynamic programming. In particular, we explain how order-theoretic fixed point theorems can be used to establish the existence of a fixed point of the Bellman operator, as well as why they are not sufficient to characterize the value function. By doing this, we present the logic behind the simple yet useful result recently obtained by Kamihigashi (Econ Theory, 2013) based on this order-theoretic approach.
\end{abstract}

Keywords Dynamic programming · Bellman equation · Value function ·

Fixed point

\section{JEL Classification C61}

\begin{abstract}
This note is based on presentations of earlier versions of Kamihigashi (2013) at various conferences and seminars including the 11th SAET Conference in Faro, 2011, the Workshop in Honor of Cuong Le Van in Exeter, 2011, a seminar at the Paris School of Economics in 2012, the 21st European Workshop on General Equilibrium Theory in Exeter, 2012, and the Asian Meeting of the Econometric Society in Singapore, 2013. I would like to thank all participants for helpful comments and suggestions. I would also like to thank an anonymous referee for bringing a related example to my attention. Financial support from the Japan Society for the Promotion of Science is gratefully acknowledged.
\end{abstract}

T. Kamihigashi $(\varangle)$

RIEB, Kobe University, Rokkodai, Nada, Kobe 657-8501, Japan

e-mail: tkamihig@rieb.kobe-u.ac.jp

T. Kamihigashi

IPAG Business School, 184 Bd Saint Germain, 75006 Paris, France 


\section{Introduction}

Dynamic programming is one of the most important tools in modern economics, especially dynamic macroeconomics. ${ }^{1}$ Recently, Kamihigashi (2013) obtained a simple yet useful result on the existence, uniqueness, and stability of a solution to the Bellman equation, i.e., a fixed point of the Bellman operator. In this note, we present the logic behind this result as well as explain why the order-theoretic approach used in Kamihigashi (2013) is useful but not sufficient to characterize the value function. To present our arguments in precise terms, we start by introducing some definitions and notations.

\section{Preliminaries}

Let $X$ be a set. Let $\Gamma$ be a nonempty-valued correspondence from $X$ to $X$. Let $D$ be the graph of $\Gamma$ :

$$
D=\{(x, y) \in X \times X: y \in \Gamma(x)\} .
$$

Let $u: D \rightarrow[-\infty, \infty)$. Let $\Pi$ and $\Pi\left(x_{0}\right)$ denote the set of feasible paths and that of feasible paths from $x_{0}$, respectively:

$$
\begin{aligned}
\Pi & =\left\{\left\{x_{t}\right\}_{t=0}^{\infty} \in X^{\infty}: \forall t \in \mathbb{Z}_{+}, x_{t+1} \in \Gamma\left(x_{t}\right)\right\}, \\
\Pi\left(x_{0}\right) & =\left\{\left\{x_{t}\right\}_{t=1}^{\infty} \in X^{\infty}:\left\{x_{t}\right\}_{t=0}^{\infty} \in \Pi\right\}, \quad x_{0} \in X .
\end{aligned}
$$

Let $\beta \in[0,1)$. Consider the following optimization problem:

$$
v^{*}\left(x_{0}\right)=\sup _{\left\{x_{t}\right\}_{t=1}^{\infty} \in \Pi\left(x_{0}\right)} \lim _{n \uparrow \infty} \sum_{t=0}^{n} \beta^{t} u\left(x_{t}, x_{t+1}\right), \quad x_{0} \in X .
$$

In this note, we assume that the above limit exists in $\overline{\mathbb{R}}$ for any feasible path $\left\{x_{t}\right\} \in \Pi .^{2}$ The function $v^{*}: X \rightarrow \overline{\mathbb{R}}$ defined in (2.4) is called the value function.

The Bellman operator $B$ on the space of functions $v: X \rightarrow \overline{\mathbb{R}}$ is defined by

$$
(B v)(x)=\sup _{y \in \Gamma(x)}\{u(x, y)+\beta v(y)\}, \quad x \in X .
$$

It should be noted that $B v$ may not be well-defined for every $v: X \rightarrow \overline{\mathbb{R}}$. In particular, $B v$ is not well-defined if there exists $(x, y) \in D$ such that

\footnotetext{
${ }^{1}$ Recent applications of dynamic programming include Algan et al. (2011), Bloch and Houy (2012), Dutta and Radner (2012), Goenka and Lin (2012), Karp and Zhang (2012), Llanes and Trento (2012), Roy and Zilcha (2012), Herrera and Martinelli (2013), Reis (2013), and Schaar et al. (2013).

2 This assumption as well as the assumption that $\beta \in[0,1)$ is made here to simplify the exposition. In Kamihigashi (2013), it is assumed that $\beta \in[0, \infty)$, and lim in (2.4) is replaced by a general limit that can be either lim inf or lim sup.
} 


$$
u(x, y)=-\infty, \quad v(y)=\infty
$$

In this case, the sum $u(x, y)+\beta v(y)$ is not well-defined; thus $B v$ is not a well-defined function.

A function $v: X \rightarrow \overline{\mathbb{R}}$ satisfying $B v=v$ is called a fixed point of $B$. At this point, two fundamental questions arise:

1. Is the value function $v^{*}$ a fixed point of the Bellman operator $B$ ?

2. Is an arbitrary fixed point of the Bellman operator $B$ equal to the value function $v^{*}$ ?

\section{Is the value function $v^{*}$ a fixed point of the Bellman operator $B$ ?}

The short answer to this question is yes with a caveat. More precisely, it follows from Kamihigashi (2008, Theorem 2) that $v^{*}$ is a fixed point of $B$ if and only if there exists no $(x, y) \in D$ such that (2.6) holds with $v=v^{*}$. If there exists such $(x, y) \in D$, then $v^{*}$ is not a fixed point of $B$, in the sense that $v^{*}$ is not even in the domain of $B$.

One way to avoid this anomaly is by assuming that

$$
\forall(x, y) \in D, \quad u(x, y)>-\infty .
$$

This assumption is used by (Stokey et al. (1989), Theorem 4.2). However, there are various economic models violating (3.1).

\subsection{An AK model with logarithmic utility}

As a simple example violating (3.1), consider the following specification:

$$
\begin{aligned}
& X=\mathbb{R}_{+}, \quad \forall x \in X, \Gamma(x)=[0, A x], \\
& \forall y \in \Gamma(x), \quad u(x, y)=\ln (A x-y),
\end{aligned}
$$

where $A>0$ is a constant. Then we have $u(x, y)=-\infty$ whenever $y=A x$.

\subsection{Discussion}

Instead of (3.1), the following condition can be used to rule out the existence of $(x, y) \in D$ satisfying (2.6) with $v=v^{*}$ :

$$
\forall x \in X, \quad v^{*}(x)<\infty
$$

This can be ensured by imposing a joint restriction on $u, \beta$, and $\Gamma$.

Alternatively, one can directly conclude from Kamihigashi (2008, Theorem 2) that as indicated above, the value function $v^{*}$ is a fixed point of the Bellman operator $B$ if and only if $B v^{*}$ is a well-defined function from $X$ to $\overline{\mathbb{R}}$. Since this is a minimum 


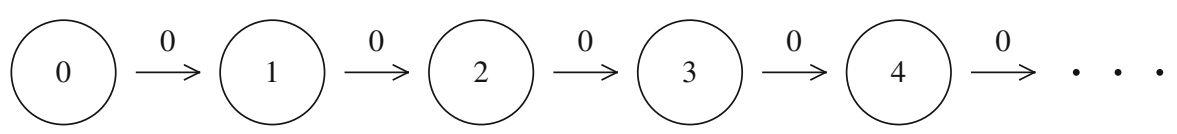

Fig. 1 Feasible transitions (arrows) and associated returns (values above arrows) under (4.1)

requirement for $v^{*}$ to be a fixed point of $B$, there is no further issue concerning the first fundamental question.

\section{Is a fixed point of the Bellman operator $B$ the value function $v^{*}$ ?}

The answer to this question is in general no. Let us illustrate this point using a simple counterexample.

\subsection{A simple counterexample}

Suppose that

$$
X=\mathbb{Z}_{+}, \quad \forall i \in X, \quad \Gamma(i)=\{i+1\}, u(i, i+1)=0 .
$$

Figure 1 shows a symbolic diagram describing this example. Note that at each state $i \in X$, there is only one feasible transition with a return of zero. Hence, given any initial state $i \in X$, one can only move to the next state $i+1$. It is important to emphasize that there is nothing pathological here: indeed, $u$ is continuous and bounded, and $\Gamma$ is continuous and compact. ${ }^{3}$

Since $u(i, i+1)=0$ for all $i \in X$, we can immediately see that

$$
\forall i \in X, \quad v^{*}(i)=0
$$

In view of (4.1), for any $i \in X$, the Bellman operator $B$ satisfies

$$
(B v)(i)=u(i, i+1)+\beta v(i+1)=\beta v(i+1) .
$$

Hence $v$ is a fixed point of $B$ if and only if

$$
\forall i \in X, \quad v(i)=\beta v(i+1) .
$$

If $v=v^{*}$, then both sides above are zero, which confirms that $v^{*}$ is a fixed point of $B$. Is there any other fixed point? To answer this, for $\alpha \in \mathbb{R}$ and $i \in X$, define

$$
v_{\alpha}(i)=\alpha \beta^{-i}
$$

3 See (Stokey et al. (1989), Section 3.3) for definitions related to correspondences. 
Then for any $\alpha \in \mathbb{R}$ and $i \in X$, we have

$$
\beta v_{\alpha}(i+1)=\beta \alpha \beta^{-(i+1)}=\alpha \beta^{-i}=v_{\alpha}(i) .
$$

Thus $v_{\alpha}$ satisfies (4.4); i.e., $v_{\alpha}$ is a fixed point of $B$. Since this is true for any $\alpha \in \mathbb{R}$, it follows that $B$ has a continuum of fixed points. Only when $\alpha=0$ does $v_{\alpha}$ coincide with the value function $v^{*} .4$

\subsection{Discussion}

The simple counterexample above suggests that for a fixed point of the Bellman operator $B$ to equal the value function, the domain of $B$ must be restricted even if $u$ is continuous and bounded and $\Gamma$ is continuous and compact. Under these conditions, $v^{*}$ is also continuous and bounded; hence it would be natural to restrict the domain of $B$ to the space of bounded continuous functions. As is well known, the Bellman operator is a contraction mapping on this space, and by the contraction mapping theorem, it has a unique fixed point in this space. Since $v^{*}$ itself is a fixed point of $B$ by (3.1) or (3.4), the unique fixed point must be the value function (Stokey et al. (1989), Theorem 4.6).

There are various extensions of this result (e.g., Durán (2000); Rinćon-Zapatero and Rodríguez-Palmero (2003, 2007, 2009); Martins-da-Rocha and Vailakis (2010), Matkowski and Nowak (2011)), most of which require the existence of a set $F$ of functions on $X$ with the following properties:

1. The Bellman operator $B$ has a fixed point in $F$.

2. Any fixed point of $B$ in $F$ equals the value function $v^{*}$.

In the case of (Stokey et al. (1989), Theorem 4.6), $F$ is the space of bounded continuous functions. As mentioned above, their approach is based on the contraction mapping theorem, which is a powerful fixed point theorem when it applies. In what follows, we propose an alternative approach based on order-theoretic fixed point theorems.

\section{An order-theoretic approach}

An inherent property of the Bellman operator $B$ that has not been fully exploited in the economic literature is its monotonicity. ${ }^{5}$ To be precise, we define the partial order $\leq$ on the set of functions from $X$ to $\overline{\mathbb{R}}$ as follows:

$$
v \leq w \Longleftrightarrow \forall x \in X, v(x) \leq w(x)
$$

It is immediate from (2.5) that $B$ is a monotone operator:

$$
v \leq w \Rightarrow B v \leq B w
$$

\footnotetext{
4 An anonymous referee has pointed out that there exists a continuum of fixed points in Example 5 of (2003) with $m=2$ and $\beta=1 / 2$, in which case $u(x, y)=-2 x+y$ and $\Gamma(x)=[0,2 x]$. It is easy to verify that in this example, any function $v(x)=a x$ with $a \geq-2$ is a fixed point of the Bellman operator.

5 See Kamihigashi (2013) for discussion of related work by Bertsekas and Shreve (1978).
} 
provided that both $B v$ and $B w$ are well-defined. If $v \leq w$, we define the order interval $[v, w]$ as the set of functions $f: X \rightarrow \overline{\mathbb{R}}$ with $v \leq f \leq w$.

There are useful fixed point theorems for monotone maps on partially ordered spaces. Among the best known are the Knaster-Tarski fixed point theorem and Tarski's fixed point theorem (Aliprantis and Border (2006), pp. 16-17).

\subsection{An application of Tarski's fixed point theorem: an initial attempt}

The following is an immediate consequence of Tarski's fixed point theorem (or the Knaster-Tarski fixed point theorem).

Lemma 5.1 Assume (3.1). Suppose that there exist functions $\underline{v}, \bar{v}: X \rightarrow \overline{\mathbb{R}}$ with $\underline{v} \leq \bar{v}$ such that B maps $[\underline{v}, \bar{v}]$ into itself. ${ }^{6}$ Then $B$ has a fixed point in $[\underline{v}, \bar{v}]$.

Recall that (3.1) ensures that $B v$ is well-defined for any $v: X \rightarrow \overline{\mathbb{R}}$. Under (3.1), if we define

$$
\underline{v}(x)=-\infty, \quad \bar{v}(x)=\infty, \quad x \in X,
$$

then $B$ trivially maps $[\underline{v}, \bar{v}]$ into itself. Hence we obtain the following result.

Proposition 5.1 Under (3.1), B has a fixed point $v: X \rightarrow \overline{\mathbb{R}}$.

Unfortunately, this result is useless. To see this, assume (5.3). Then

$$
\forall x \in X, \quad(B \underline{v})(x)=\sup _{y \in \Gamma(x)}\{u(x, y)+\beta \cdot(-\infty)\}=-\infty .
$$

Hence $B \underline{v}=\underline{v}$. We similarly obtain $B \bar{v}=\bar{v}$. Since these trivial functions are always fixed points of $B$, Proposition 5.1 offers no additional information.

\subsection{An application of Tarski's fixed point theorem: a second attempt}

We can avoid the trivial fixed points discussed above if we require $\underline{v}$ and $\bar{v}$ in Lemma 5.1 to be finite-valued, in which case any fixed point of $B$ in $[\underline{v}, \bar{v}]$ is finite-valued. The following result is once again an immediate consequence of Tarski's fixed point theorem (or the Knaster-Tarski fixed point theorem).

Proposition 5.2 Suppose that there exist functions $\underline{v}, \bar{v}: X \rightarrow \mathbb{R}$ with $\underline{v} \leq \bar{v}$ such that $B$ maps $[\underline{v}, \bar{v}]$ into itself. Then $B$ has a fixed point in $[\underline{v}, \bar{v}]$.

Unlike Lemma 5.1, this result does not require (3.1) since for any $(x, y) \in D$ and $v: X \rightarrow \mathbb{R}$, the sum $u(x, y)+\beta v(y)$ is well-defined, which implies that $B v$ is well-defined. ${ }^{7}$ Since both $\underline{v}$ and $\bar{v}$ are finite-valued, any fixed point of $B$ in $[\underline{v}, \bar{v}]$ is a finite-valued function. Does such a fixed point equal the value function $v^{*}$ ?

\footnotetext{
6 Since $B$ is monotone, $B$ maps $[\underline{v}, \bar{v}]$ into itself if and only if $\underline{v} \leq B \underline{v}$ and $B \bar{v} \leq \bar{v}$.

7 In fact, $B v$ is well-defined as long as $v: X \rightarrow \mathbb{R} \cup\{-\infty\}$.
} 
The answer is once again no in general. To see this, consider the counterexample in Sect. 4.1. Recall the definition of $v_{\alpha}$ in (4.5). Let $\underline{v}=v_{-1}$ and $\bar{v}=v_{1}$. Then $\underline{v}$ and $\bar{v}$ are finite-valued and, in addition, fixed points of $B$. Thus $B$ maps $[\underline{v}, \bar{v}]$ into itself, and by Proposition 5.2, $B$ has a fixed point in $[\underline{v}, \bar{v}]$. However, this result provides no additional information since both $\underline{v}$ and $\bar{v}$ are already fixed points of $B$. In fact, it follows from (4.6) that $B$ has a continuum of fixed points in $[\underline{v}, \bar{v}]$, each of which is a finite-valued function, but only one of them is the value function.

\section{Additional conditions on $\underline{v}$ and $\bar{v}$}

The discussion in the previous section indicates that for an arbitrary fixed point of $B$ in $[\underline{v}, \bar{v}]$ to equal the value function $v^{*}$, we need additional conditions on $\underline{v}$ and $\bar{v}$ even when both are finite-valued functions. To identify such conditions, it is useful to note that finite iterations of the Bellman operator are equivalent to finite-horizon approximations of the original infinite-horizon problem. More precisely, $B^{n} v$ is the value function of the $n$-period problem with the value of the terminal stock evaluated by the function $v$ :

Lemma 6.1 [Kamihigashi (2013), Lemma A.2] Suppose that there exist functions $\underline{v}, \bar{v}: X \rightarrow \mathbb{R} \cup\{-\infty\}$ with $\underline{v} \leq \bar{v}$ such that $B$ maps $[\underline{v}, \bar{v}]$ into itself. Let $v \in[\underline{v}, \bar{v}]$. Then for any $n \in \mathbb{N}$ and $x_{0} \in X$, we have

$$
\left(B^{n} v\right)\left(x_{0}\right)=\sup _{\left\{x_{t}\right\}_{t=1}^{\infty} \in \Pi\left(x_{0}\right)}\left\{\sum_{t=0}^{n-1} \beta^{t} u\left(x_{t}, x_{t+1}\right)+\beta^{n} v\left(x_{n}\right)\right\} .
$$

Recalling the definition of the value function $v^{*}$ in (2.4), one may conjecture that for $v$ to equal $v^{*}$, the "extra" term $\beta^{n} v\left(x_{n}\right)$ should disappear asymptotically. In fact, it is shown in Stokey et al. (1989, Theorem 4.3) that under (3.1), a fixed point of $B$ satisfying the following condition is the value function $v^{*}$ :

$$
\forall\left\{x_{t}\right\}_{t=0}^{\infty} \in \Pi, \quad \lim _{t \uparrow \infty} \beta^{t} v\left(x_{t}\right)=0 .
$$

As a simple application of this condition, consider the continuum of fixed points constructed in Sect. 4.1. Note from (4.1), (4.4), and (4.5) that for any $\alpha \in \mathbb{R}, t \in \mathbb{N}$, and feasible path $\left\{x_{t}\right\}$, we have

$$
\beta^{t} v_{\alpha}\left(x_{t}\right)=\beta^{t-1} v_{\alpha}\left(x_{t-1}\right)=\cdots=v_{\alpha}\left(x_{0}\right)=\alpha \beta^{-x_{0}} .
$$

Therefore, among the continuum of fixed points $v_{\alpha}$ of $B$, only the value function $v^{*}=v_{0}$ satisfies (6.2), as expected.

Although a fixed point of $B$ satisfying (6.2) is guaranteed to be the value function under (3.1), the value function itself may not satisfy (6.2). For example, in the AK model of Sect. 3.1, we have $v^{*}(0)=-\infty$; thus $v^{*}$ does not satisfy (6.2) for the feasible path identically equal to zero. 
To handle such cases, it is useful to decompose the limit condition in (6.2) into two parts:

$$
\begin{gathered}
\liminf _{t \uparrow \infty} \beta^{t} v\left(x_{t}\right) \geq 0, \\
\limsup _{t \uparrow \infty} \beta^{t} v\left(x_{t}\right) \leq 0 .
\end{gathered}
$$

Consider again the AK model of Sect. 3.1. It can easily be shown that (6.5) with $v=v^{*}$ is satisfied for any feasible path. Hence (6.5) is a property of the value function $v^{*}$. In contrast, (6.4) with $v=v^{*}$ is violated not only for the path identically equal to zero but also for feasible paths converging to zero sufficiently fast. Therefore, the value function $v^{*}$ satisfies (6.4) only for reasonably "good" paths. It turns out that such paths are provided by the following set:

$$
\Pi^{0}=\left\{\left\{x_{t}\right\} \in \Pi: \lim _{n \uparrow \infty} \sum_{t=0}^{n} \beta^{t} u\left(x_{t}, x_{t+1}\right)>-\infty\right\} .
$$

The idea of requiring (6.5) only for the paths in $\Pi^{0}$ is due to Le Van and Morhaim (2002).

The preceding discussion suggests the following conditions:

$$
\begin{aligned}
& \forall\left\{x_{t}\right\}_{t=0}^{\infty} \in \Pi^{0}, \quad \liminf _{t \uparrow \infty} \beta^{t} \underline{v}\left(x_{t}\right) \geq 0, \\
& \forall\left\{x_{t}\right\}_{t=0}^{\infty} \in \Pi, \quad \limsup _{t \uparrow \infty} \beta^{t} \bar{v}\left(x_{t}\right) \leq 0 .
\end{aligned}
$$

It can be shown that given any fixed point $v$ of $B$ in $[\underline{v}, \bar{v}]$, if $v$ satisfies (6.7), then $v \geq v^{*}$, and if $v$ satisfies (6.8), then $v \leq v^{*}$; see Kamihigashi (2013) and Stokey et al. (1989, Theorem 4.3). Adding these requirements to Proposition 5.2 and recalling footnote 7, we obtain parts (a) and (b) of the following result.

Theorem 6.1 [Kamihigashi (2013), Theorem 2.1] Suppose that there exist functions $\underline{v}, \bar{v}: X \rightarrow \mathbb{R} \cup\{-\infty\}$ with $\underline{v} \leq \bar{v}$ such that $B$ maps $[\underline{v}, \bar{v}]$ into itself and such that (6.7) and (6.8) hold. Then the following conclusions hold:

(a) The Bellman operator $B$ has a unique fixed point in $[\underline{v}, \bar{v}]$.

(b) This fixed point is the value function $v^{*}$.

(c) The increasing sequence $\left\{B^{n} \underline{v}\right\}_{n=1}^{\infty}$ converges to $v^{*}$ pointwise.

As in Lemma 5.1 and Proposition 5.2, $B$ has a fixed point in $[\underline{v}, \bar{v}]$ by Tarski's fixed point theorem (or the Knaster-Tarski fixed point theorem). By (6.7) and (6.8), any fixed point of $B$ in $[\underline{v}, \bar{v}]$ is the value function $v^{*}$. This in turn implies that $B$ has only one fixed point in $[\underline{v}, \bar{v}]$. Hence conclusions (a) and (b) follow.

Conclusion (c) is stated here only for the reader's convenience. See Kamihigashi (2013) for discussion of this and other aspects of the above result. 


\section{References}

Aliprantis, C.D., Border, K.C.: Infinite Dimensional Analysis: A Hitchhiker's Guide, 3rd edn. Springer, Berlin (2006)

Algan, Y., Challe, E., Ragot, X.: Incomplete markets and the output-inflation tradeoff. Econ. Theory 46, 55-84 (2011)

Bertsekas, D.P., Shreve, S.E.: Stochastic Optimal Control: The Discrete Time Case. Academic Press, New York (1978)

Bloch, F., Houy, N.: Optimal assignment of durable objects to successive agents. Econ. Theory 51, 13-33 (2012)

Durán, J.: On dynamic programming with unbounded returns. Econ. Theory 15, 339-352 (2000)

Dutta, P.K., Radner, R.: Capital growth in a global warming model: will China and India sign a climate treaty? Econ. Theory 49, 411-443 (2012)

Goenka, A., Lin, L.: Infectious diseases and endogenous fluctuations. Econ. Theory 50, 125-149 (2012)

Herrera, H., Martinelli, C.: Oligarchy, democracy, and state capacity. Econ. Theory 52, 165-186 (2013)

Kamihigashi, T.: On the principle of optimality for nonstationary deterministic dynamic programming. Int. J. Econ. Theory 4, 519-525 (2008)

Kamihigashi, T.: Elementary results on solutions to the Bellman equation of dynamic programming: existence, uniqueness, and convergence. Econ. Theory (2013). doi:10.1007/s00199-013-0789-4

Karp, L., Zhang, J.: Taxes versus quantities for a stock pollutant with endogenous abatement costs and asymmetric information. Econ. Theory 49, 371-409 (2012)

Le Van, C., Morhaim, L.: Optimal growth models with bounded or unbounded returns: a unifying approach. J. Econ. Theory 105, 158-187 (2002)

Llanes, G., Trento, S.: Paten policy, patent pools, and the accumulation of claims in sequential innovation. Econ. Theory 50, 703-725 (2012)

Martins-da-Rocha, V.F., Vailakis, Y.: Existence and uniqueness of a fixed point for local contractions. Econometrica 78, 1127-1141 (2010)

Matkowski, J., Nowak, A.S.: On discounted dynamic programming with unbounded returns. Econ. Theory 46, 455-474 (2011)

Reis, C.: Taxation without commitment. Econ. Theory 52, 565-588 (2013)

Rinćon-Zapatero, J.P., Rodríguez-Palmero, C.: Existence and uniqueness of solutions to the Bellman equation in the unbounded case. Econometrica 71, 1519-1555 (2003)

Rinćon-Zapatero, J.P., Rodríguez-Palmero, C.: Recursive utility with unbounded aggregators. Econ. Theory 33, 381-391 (2007)

Rinćon-Zapatero, J.P., Rodríguez-Palmero, C.: Corrigendum to "Existence and uniqueness of solutions to the Bellman equation in the unbounded case" Econometrica, Vol. 71, No. 5 (September, 2003), 15191555. Econometrica 77, 317-318 (2009)

Roy, S., Zilcha, I.: Stochastic growth with short-run prediction of shocks. Econ. Theory 51, 539-580 (2012)

Stokey, N., Lucas Jr, R.E.: Recursive Methods in Economic Dynamics. Harvard University Press, Cambridge (1989)

van der Schaar, M., Xu, J., Zame, W.: Efficient online exchange via fiat money. Econ. Theory 54, 211-248 (2013) 